\title{
Wild Edible Plants of Savaştepe District (Balıkesir, Turkey)
}

\author{
Ebru Özdemir, Şükran Kültür
}

\begin{abstract}
This ethnobotanical study was planned to carried out in villages of Savaştepe district (Balıkesir, Turkey) between May 2012 to October 2015. The settlements (44 villages) in Savaştepe were visited during the field work. With this study, we aimed to document traditional uses of edible plants in Savaştepe (Balıkesir). Interviews were made with the local people. A total of 205 individuals ( 107 men, 98 women) interviewed in the area. The plant samples were collected in company with local people. The collected specimens were identified and were deposited as
\end{abstract}

herbarium samples at ISTE (The Herbarium of the Faculty of Pharmacy of Istanbul University). Among these collected plants 71 taxa belonging to 27 families are edible plants. The plants were mostly used as food (55 taxa), spice (14 taxa), beverage (3 taxa) and honey paste (2 taxa). Mostly used parts of plants were leaf (27 taxa), aerial part (20 taxa), fruit (19 taxa) and seed (5 taxa).

Keywords: Ethnobotany, Wild edible plants, Savaştepe, Balıkesir, Turkey
Ebru Özdemir

Istanbul Yeni Yuzyil University, Faculty of Pharmacy, Department of Pharmaceutical Botany, 34010, Istanbul, TURKEY

Şükran Kültür

Istanbul University, Faculty of Pharmacy, Department of Pharmaceutical Botany, 34116, Istanbul, TURKEY

Corresponding Author:

Ebru Özdemir Nath

e-mail:pharmebru@gmail.com

Submitted / Gönderilme: 24.01.2017

Accepted / Kabul: 18.04.2017
Revised / Düzeltme: 18.04.2017

\section{Introduction}

Turkey's geographical location is at the meeting point of three phytogeographical regions (Euro-Siberian, Mediterranean and lrano-Turanian). Because of this, Anatolia has a very rich flora. The flora of Turkey is estimated to contain approximately 9996 species of vascular plants, one third of its flora $(33.3 \%)$ is endemic (1). Study area, Savaştepe, is a district of Balıkesir province in the Marmara region of Turkey (Figure 1). It has an area of $430 \mathrm{~km}$. Its plant diversity is very rich because of its localization meeting point of 2 phytogeographic regions (Mediterranean, Euro-Siberian). The population is 20.201. Savaştepe has 44 villages. Nomadic culture is seen in the villages of Savaştepe. Karakeçili, Hardal, Kubaş, Kılaz, Yüncü yoruks live in the villages of Savaştepe. Karakeçili Yoruk communities live in 20 villages, Hardal yoruk communities live in 6 villages, Kubaş yoruk communities live in 10 villages, Kilaz yoruk communities live in 2 villages, Yüncü yoruk communities live in 1 village. This study records the wild edible plants of Savaştepe (Balıkesir) for the first time. 


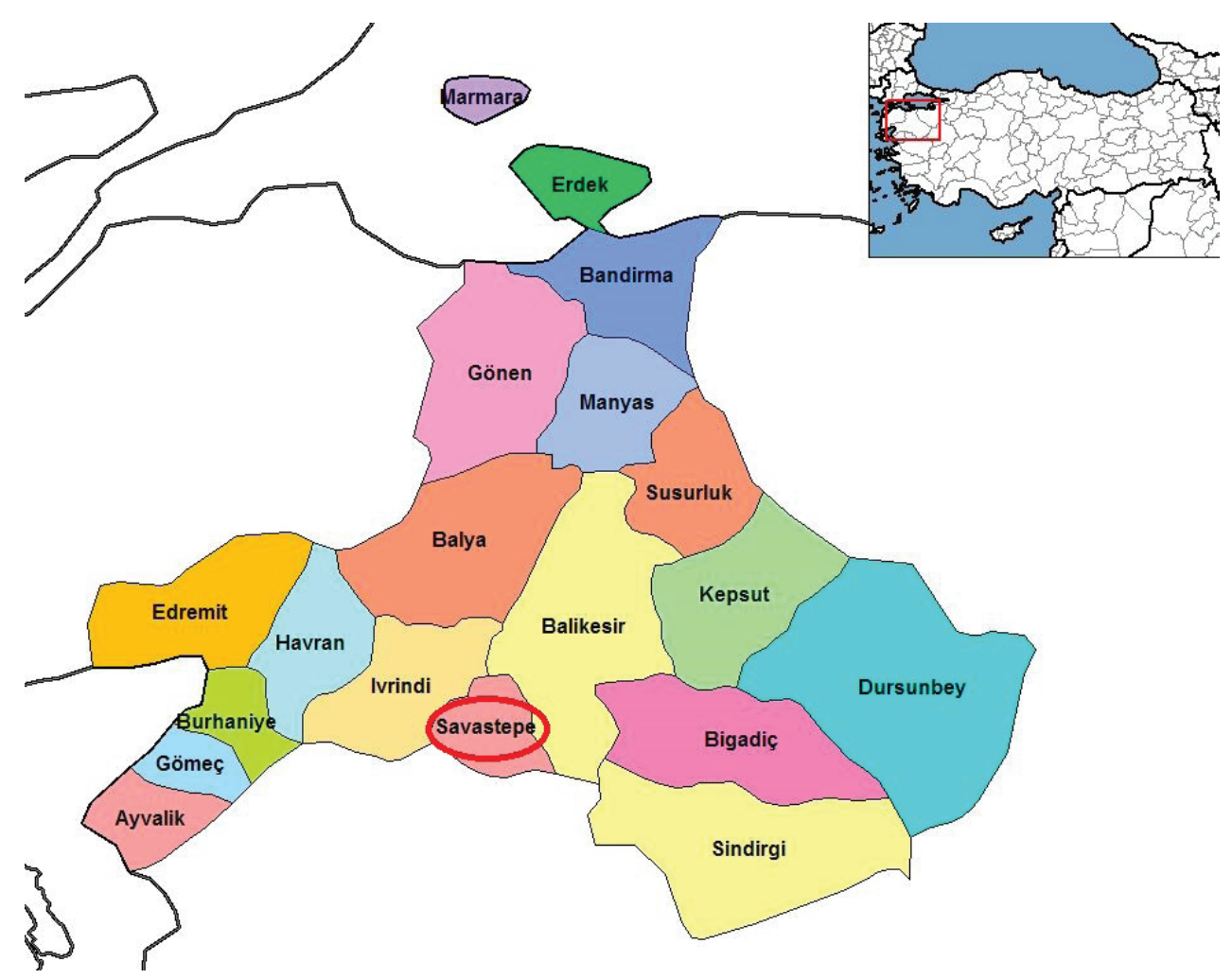

Figure 1. The map of Savaştepe, Balıkesir and Turkey

\section{Material and Methods}

This study was conducted between May 2012 to October 2015. The settlements in Savaştepe (44 villages) were visited during the field work (2). The information including the various data such as local names, part of the used plants, preparation and cooking methods were obtained by mean of direct interviews with villagers who know practice about the useful plants. A total of 205 individuals (107 men, 98 women) interviewed in the area (Figures 2-5). During the

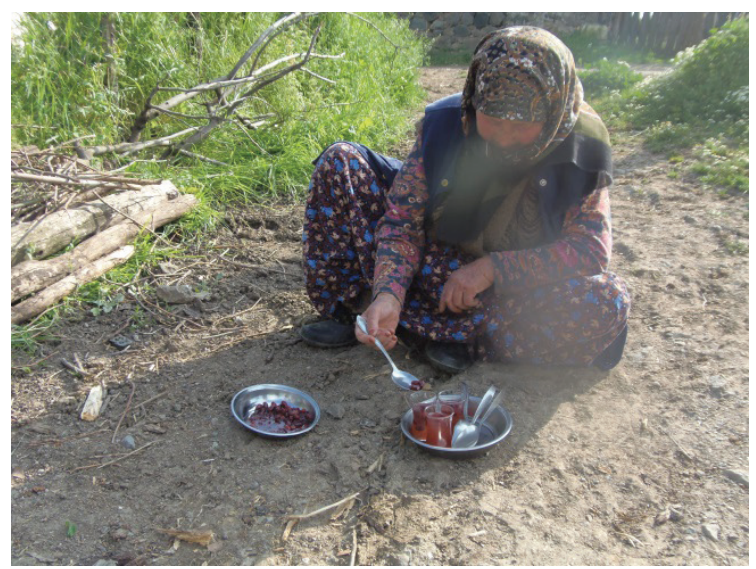

Figure 2. A villager is serving Cornus mas (Kizılcık) compote in Çaltılı village, Savaştepe. field studies, the plant specimens were collected together with accompanied informants. The collected fresh materials were numbered and kept as herbarium samples at ISTE (Herbarium of Faculty of Pharmacy, Istanbul University) for botanical identification. Some plant species deposited as a personel collection with the code of E.Ö. The collected specimens were identified by using "Flora of Turkey and the East Aegean Islands" (3-5) and were compared with the specimens deposited at ISTE. Plant names reviewed according to the literature $(1,6)$.

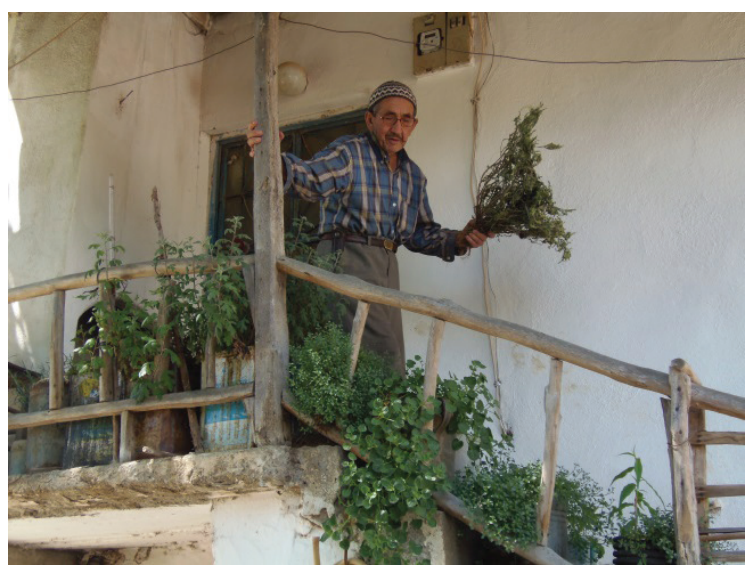

Figure 3. A villager with Cnicus benedictus in Kovukyurt village, Savaştepe. 


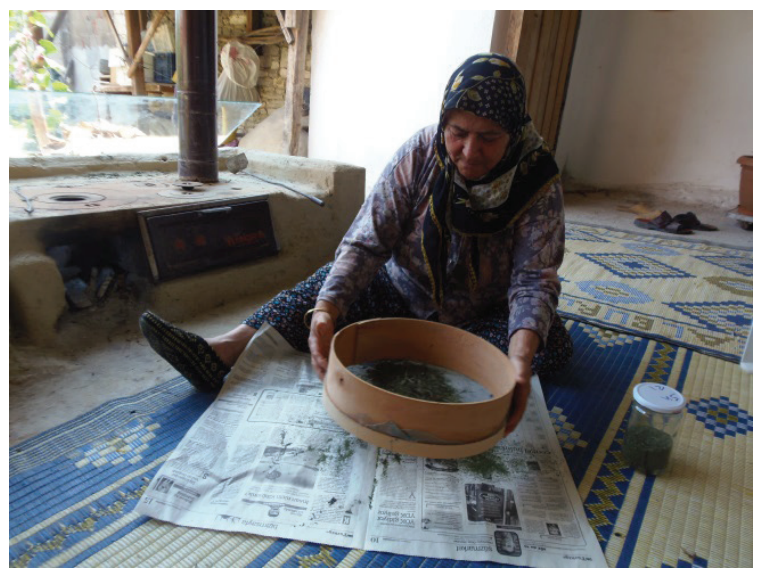

Figure 4. A villager is making spice with Mentha longifolia (L.) L. subsp. typhoides (Nane) in Karaçam village, Saveştepe.

\section{Results and Discussion}

This study is the first ethnobotanical study to determine the wild edible plants of Savaştepe district. 205 people were interviewed in this study. Following the identification of the specimens in ISTE (Herbarium of Istanbul University, Faculty of Pharmacy), 71 taxa belonging to 27 families are edible plants (Table 1). The plants were mostly used as food (55 taxa), spice ( 14 taxa), beverage ( 3 taxa) and honey paste ( 2 taxa). Mostly used parts of plants were leaf (27 taxa), aerial part (20 taxa), fruit (19 taxa) and seed (5 taxa). The most frequently used families are Rosaceae (16\%), Compositae (14\%) and Lamiaceae (11\%) (Figure 6). Among the edible plants of Savaştepe, the genus that contains the most taxa are: Allium L., Convolvulus L., Crataegus L., Prunus L., Pyrus L., Rosa L., Rubus L., Rumex L., Thymus L. and Urtica L..

The results of this study compared to the findings of other studies carried out in Marmara region of Turkey, show there are some new usages of plants recorded. Among these 71 taxa, 43 taxa have been recorded as edible plant in Marmara region before (Table 2), 28 taxa have been recorded as edible plant in Balıkesir for the first time (7-16). According to the other studies in Marmara region these 43 taxa have common uses in the region and because of that they may have high economic value and the local people can be informed to cultivate these plants. The most commonly used edible taxa with at least 5 regions in 10 studies are; Cornus mas L., Crataegus monogyna Jacq., Erodium cicutarium (L.) L'Hér., Malva sylvestris L., Morus nigra L., Pistacia terebinthus L., Polygonum cognatum

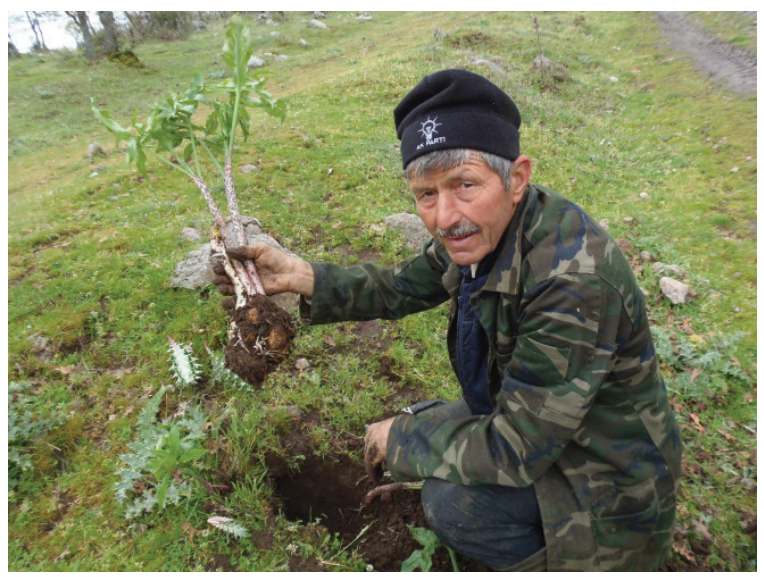

Figure 5. A villager is collecting Arum elongatum Steven root for cooking in Karapınar village, Savaştepe.

Meisn., Prunus divaricata Ledeb. subsp. divaricata (Ledeb.) Schneider, Prunus spinosa L., Pyrus amygdaliformis Vill. Pyrus elaeagnifolia Pall., Rosa canina L., Rubus canescens DC., Rubus sanctus Schreb., Rumex acetosella L., Rumex patientia L., Sonchus asper (L.) Hill subsp. glaucescens (Jord.) Ball ex Ball, Urtica dioica L.

The importance of food in medicine was recognized in the 5th Century BC by Hippocrates, who is considered the father of western medicine. The phrase "Let food be your medicine and medicine be your food", a widespread phrase quoted by today's scientists. According to the results of that study some edible plants have been widely consumed as a medicine in Savaştepe (17). For example; the fruit of Ruscus aculeatus L. was freshly eaten for heart problems, the root of Cnicus benedictus L. was cooked with onion and eaten for allergy, the fruit of Arbutus andrachne L. was freshly eaten for diabetes, the leaf of Rumex crispus L. was cooked and eaten for urinary tract inflamation. The use of wild edible plants is generally widespread among elderly people traditionally and in danger of vanishing in the future. Because of that local and government authorities should give importance to protect traditional knowledge. It is important to record the genetic diversity of food plants and to prevent genetic erosion and may contribute to find solutions for a progressively impoverished agriculture. Wealth of natural vegetable resources help to develop new agricultural production. Finally, this kind of applied botanical research helps to preserve cultural heritage through multidisciplinary approach of the ethnobotany. 
Table 1: Wild edible plants of Savaştepe (Balıkesir-Turkey)

\begin{tabular}{|c|c|c|c|}
\hline Botanical name, family and specimen number & Local name in Savaştepe & Used parts & / Usage \\
\hline \multicolumn{4}{|l|}{ SPERMATOPHYTA GYMNOSPERMAE } \\
\hline \multicolumn{4}{|l|}{ Pinaceae } \\
\hline Pinus brutia Ten. (ISTE 109873) & Çam & Resin & Mixed with honey and eaten \\
\hline \multicolumn{4}{|l|}{ SPERMATOPHYTA ANGIOSPERMAE } \\
\hline \multicolumn{4}{|l|}{ Amaranthaceae } \\
\hline Chenopodium murale L. (ISTE 109535) & Deli Kazayağı, Ekşigüney & Leaf & $\begin{array}{l}\text { Cooked as a meal } \\
\text { Eaten fresh } \\
\text { Used in pastry }\end{array}$ \\
\hline \multicolumn{4}{|l|}{ Amaryllidaceae } \\
\hline Allium paniculatum L. (ISTE 109756, ISTE 109758) & Yoğurtçuk otu & $\begin{array}{l}\text { Aerial part } \\
\text { Leaf }\end{array}$ & $\begin{array}{l}\text { Cooked as a meal with egg } \\
\text { Eaten fresh }\end{array}$ \\
\hline \multicolumn{4}{|l|}{ Anacardiaceae } \\
\hline Pistacia terebinthus L. (ISTE 10536, 109537) & Çertlemik, Çetek & $\begin{array}{l}\text { Dry fruit } \\
\text { Young leaf } \\
\text { Oil (From fruit) } \\
\text { Fruit }\end{array}$ & $\begin{array}{l}\text { As coffea } \\
\text { Eaten fresh } \\
\text { Used to cook pastry } \\
\text { Eaten } \\
\text { Pickle }\end{array}$ \\
\hline Rhus coriaria L. (ISTE 109538, 109540, 109539) & Somak, Somak otu, Sumak & $\begin{array}{l}\text { Fruit, seed } \\
\text { Seed } \\
\text { Fruit, stem }\end{array}$ & $\begin{array}{l}\text { Spice } \\
\text { Powdered and used in pastry } \\
\text { Used in vinegar, pickle }\end{array}$ \\
\hline \multicolumn{4}{|l|}{ Apiaceae } \\
\hline Anethum graveolens L. (ISTE 109551, 109550) & Arapsaçı, Çarşır, Kırca, Tere & Leaf & Spice \\
\hline Foeniculum vulgare Mill. (E.Ö.6) & Arap saçı, Rezene & Leaf & Spice \\
\hline Laser trilobum (L.) Borkh. (ISTE 109552) & Yabani kimyon & Root, fruit & Spice \\
\hline \multicolumn{4}{|l|}{ Araceae } \\
\hline Arum elongatum Steven (ISTE 109559, 109560) & El kabartan, Gavurotu, Kabarcık, Kabarağ & $\begin{array}{l}\text { Root, skapus } \\
\text { Drid leaf } \\
\end{array}$ & $\begin{array}{l}\text { After the first boiling water has } \\
\text { been dumped, cooked as a meal } \\
\text { Cooked as a meal }\end{array}$ \\
\hline $\begin{array}{l}\text { Dracunculus vulgaris Schott (ISTE 109554, 109555, } \\
109556,109557,109558)\end{array}$ & El kabartan, Gavurotu, Kabarcık, Yılancık & Root & $\begin{array}{l}\text { After the first boiling water has } \\
\text { been dumped, cooked as a meal }\end{array}$ \\
\hline \multicolumn{4}{|l|}{ Asparagaceae } \\
\hline Ruscus aculeatus L. (ISTE 109562) & $\begin{array}{l}\begin{array}{l}\text { Değirmen boncuğu, Deve çöküren, Deve } \\
\text { tomurcuğu }\end{array} \\
\end{array}$ & Fruit & Eaten \\
\hline \multicolumn{4}{|l|}{ Brassicaceae } \\
\hline Alyssum murale Waldst. \& Kit. (ISTE 109604, 109605) & Sünnetlice & Aerial part & Spice \\
\hline Brassica nigra (L.) K.Koch (ISTE 109608) & Hardala & Seed & $\begin{array}{l}\text { Powdered and mixed with honey, } \\
\text { eaten }\end{array}$ \\
\hline Capsella bursa-pastoris (L.) Medik. (ISTE 109606) & Çoban çantası & Aerial part & Cooked as a meal \\
\hline Lepidium spinosum Ard. (E.Ö.24) & Muşurat & Aerial part & $\begin{array}{l}\text { Freshly eaten } \\
\text { As a salad, eaten } \\
\text { Cooked as a meal }\end{array}$ \\
\hline Rapistrum rugosum (L.) All. (ISTE 109609, 109600) & Hardala, Hardal otu & Leaf & $\begin{array}{l}\text { Cooked with egg, as a omlet } \\
\text { Freshly eaten }\end{array}$ \\
\hline \multicolumn{4}{|l|}{ Compositae } \\
\hline Carduus nutans L. (ISTE 109620) & Deve dikeni & Seed & Eaten \\
\hline Carthamus lanatus L. (ISTE 109640) & Dikenli afin, Dikenli afyon & Oil (From fruit) & Used to cook pastry \\
\hline Chondrilla juncea L. (E.Ö.14, ISTE 109638) & Sakızlık & Aerial part & Cooked as a meal \\
\hline Cnicus benedictus L. (ISTE 109621, 109616) & Acı dürlek, Diken, Diken otu & Aerial part & Cooked as a meal \\
\hline Lactuca serriola L. (E.Ö.16) & Helvacık & Aerial part & Freshly eaten \\
\hline
\end{tabular}




\begin{tabular}{|c|c|c|c|}
\hline Botanical name, family and specimen number & Local name in Savaştepe & Used parts & / Usage \\
\hline $\begin{array}{l}\text { Leontodon tuberosus L. (ISTE 109636, 109637, } \\
\text { 109635) }\end{array}$ & Hindibağ, Karahindibağ & Leaf & $\begin{array}{l}\text { As a salad eaten } \\
\text { Cooked as a meal }\end{array}$ \\
\hline Silybum marianum (L.) Gaertn. (ISTE 109639) & Çıtırgan, Deve dikeni, Kenger, Sarıkız & \begin{tabular}{|l|} 
Seed \\
Latex \\
Inside of the stem \\
\end{tabular} & $\begin{array}{l}\text { Eaten } \\
\text { Spice } \\
\text { Eaten }\end{array}$ \\
\hline $\begin{array}{l}\text { Sonchus asper (L.) Hill subsp. glaucescens (Jord.) Ball } \\
\text { ex Ball (ISTE 109630, 109633, 109634, 109627) }\end{array}$ & $\begin{array}{l}\text { Diken otu, Eşek dikeni, Eşek helvası, } \\
\text { Helvacık }\end{array}$ & Aerial part & $\begin{array}{l}\text { Cooked as a meal } \\
\text { Freshly eaten }\end{array}$ \\
\hline Taraxacum hybernum Steven (ISTE 109641) & Karahindibağ, Radika, Üfleme otu & Leaf & $\begin{array}{l}\text { Cooked as a meal } \\
\text { Freshly eaten as a salad }\end{array}$ \\
\hline $\begin{array}{l}\text { Urospermum picroides (L.) Scop. ex F.W.Schmidt } \\
\text { (ISTE 109629) }\end{array}$ & Helvacik & Leaf & Cooked as a meal \\
\hline \multicolumn{4}{|l|}{ Convolvulaceae } \\
\hline Convolvulus arvensis L. (ISTE 109670, 109669) & Filiz, Sarmaşık & Aerial part & Cooked as a meal \\
\hline Convolvulus scammonia L. (ISTE 109668) & Sarmaşık & Leaf & $\begin{array}{l}\text { Mixed with cheese, Used to cook } \\
\text { pastry }\end{array}$ \\
\hline \multicolumn{4}{|l|}{ Cornaceae } \\
\hline Cornus mas L. (ISTE 109595, 109594) & Kızılcık, Küren & Fruit & Jam, compote \\
\hline \multicolumn{4}{|l|}{ Dioscoreaceae } \\
\hline $\begin{array}{l}\text { Dioscorea communis (L.) Caddick \& Wilkin (ISTE } \\
\text { 109676, 109675, 109673, 109677, 109674) }\end{array}$ & $\begin{array}{l}\text { Acı filiz, Acı ot, Adem otu, Akıllı sarmaşık, } \\
\text { Çıtırga filizi }\end{array}$ & Young shoot & $\begin{array}{l}\text { Cooked with egg, as a omlet } \\
\text { Freshly eaten }\end{array}$ \\
\hline \multicolumn{4}{|l|}{ Ericaceae } \\
\hline Arbutus andrachne L. (ISTE 109679, 109678) & $\begin{array}{l}\text { Ağaç çileği, Ayı üzümü, Dağ çileği, } \\
\text { Davulgu }\end{array}$ & Fruit & $\begin{array}{l}\text { Eaten } \\
\text { Jam, molasses }\end{array}$ \\
\hline \multicolumn{4}{|l|}{ Fagaceae } \\
\hline $\begin{array}{l}\text { Quercus infectoria G.Olivier (ISTE 109688, 109693, } \\
\text { 109694, 109692) }\end{array}$ & Akgobak, Akmeşe, Akpıynar & Fruit & As coffea \\
\hline \multicolumn{4}{|l|}{ Geraniaceae } \\
\hline Erodium ciconium (L.) L’Hér. (ISTE 109701) & Hıdır otu, Mor çiçek & Leaf & Cooked as a meal \\
\hline Erodium cicutarium (L.) L'Hér. (ISTE 109700) & Kocakarı iğnesi & Aerial part & Freshly eaten as a salad \\
\hline \multicolumn{4}{|l|}{ Lamiaceae } \\
\hline $\begin{array}{l}\text { Mentha longifolia (L.) subsp. typhoides (Briq.) Harley } \\
\text { (ISTE 109834) }\end{array}$ & Dere nanesi, Köpek nanesi & Leaf & Spice \\
\hline $\begin{array}{l}\text { Micromeria myrtifolia Boiss. \& Hohen. (ISTE 109836, } \\
\text { 109835, 109837) }\end{array}$ & Ayaklı kekik & Aerial part & Spice \\
\hline Ocimum basilicum L. (E.Ö.37) & Fesleğen, Reyhan & Leaf & Spice \\
\hline $\begin{array}{l}\text { Origanum vulgare L. subsp. hirtum (Link) Ietsw. } \\
\text { (ISTE 109829, 109814, 109813) }\end{array}$ & Akkekik, Ayaklı kekik, Dal kekiği & Aerial part & $\begin{array}{l}\text { Mixed with cheese, Used to cook } \\
\text { pastry } \\
\text { Spice }\end{array}$ \\
\hline Rosmarinus officinalis L. (ISTE 109838, 109839) & Akıl otu, Biberiye & Leaf & Spice \\
\hline Salvia napifolia Jacq. (ISTE 109795) & Çay otu & Aerial part & As a tea \\
\hline $\begin{array}{l}\text { Thymus longicaulis C.Presl subsp. chaubardii (Rchb.f.) } \\
\text { Jalas (ISTE 109832) }\end{array}$ & Akbaşlı ot, Güve otu, Kekik & Aerial part & Spice \\
\hline $\begin{array}{l}\text { Thymus zygioides Griseb. (ISTE 109819, 109818, } \\
\text { 109817, 109816, 109815, 109775) }\end{array}$ & Bayır çayı, Kaya kekiği, Kekik & Leaf & Spice \\
\hline \multicolumn{4}{|l|}{ Lauraceae } \\
\hline Laurus nobilis L. (ISTE 109855) & Defne & Leaf & Spice \\
\hline
\end{tabular}




\begin{tabular}{|c|c|c|c|}
\hline Botanical name, family and specimen number & Local name in Savaştepe & Used parts & / Usage \\
\hline \multicolumn{4}{|l|}{ Leguminosae } \\
\hline Cercis siliquastrum L. (ISTE 109733) & Erguvan, Keçi gevişi, Yabani keçiboynuzu & Flower & Eaten \\
\hline Vicia villosa Roth (ISTE 109747, 109748, 109749) & Bağla otu, Deli bakla, Fi, Yabani bezelye & Aerial part & As a salad eaten \\
\hline \multicolumn{4}{|l|}{ Malvaceae } \\
\hline Malva sylvestris L. (ISTE 109767, 109769, 109768) & Develik otu, Ebegümeci, Evelik, Gömeç & Leaf & $\begin{array}{l}\text { Cooked as a meal } \\
\text { Cooked with egg, as a omlet } \\
\text { Mixed with cheese, Used to cook } \\
\text { pastry }\end{array}$ \\
\hline \multicolumn{4}{|l|}{ Moraceae } \\
\hline Ficus carica L. (ISTE 109771) & İncir & Fruit & Jam \\
\hline Morus nigra L. (E.Ö.40) & Kara dut & Fruit & $\begin{array}{l}\text { Jam } \\
\text { Eaten } \\
\text { Sherbet for salads }\end{array}$ \\
\hline \multicolumn{4}{|l|}{ Papaveraceae } \\
\hline Papaver somniferum L. (ISTE 109867) & Afyon & Seed oil & Used for cooking \\
\hline \multicolumn{4}{|l|}{ Platanaceae } \\
\hline Platanus orientalis L. (ISTE 109877) & Çınar & Leaf & $\begin{array}{l}\text { Dried and powdered, eaten with } \\
\text { rice as a spice }\end{array}$ \\
\hline \multicolumn{4}{|l|}{ Polygonaceae } \\
\hline Polygonum cognatum Meisn. (ISTE 109880) & Madimak, Tavuk otu & Aerial part & $\begin{array}{l}\text { Cooked as a meal } \\
\text { Used to cook pastry }\end{array}$ \\
\hline Rumex acetosella L. (E.Ö.44) & Acı kulak, Ekşi kulak, Epelek & $\begin{array}{l}\text { Seed } \\
\text { Leaf }\end{array}$ & $\begin{array}{l}\text { Eaten } \\
\text { Freshly eaten as a salad } \\
\text { Cooked as a meal }\end{array}$ \\
\hline Rumex crispus L. (ISTE 109883, 109884) & $\begin{array}{l}\text { Alabardağı, Ebe kuzulağı, Eşek alabadası, } \\
\text { Labada }\end{array}$ & Leaf & Cooked as a meal \\
\hline Rumex obtusifolius L. (ISTE 109879) & Alabardak & Leaf & Freshly eaten as a salad \\
\hline Rumex patientia L. (E.Ö. 45, 46, 47) & Labada otu, Pazı & Leaf & $\begin{array}{l}\text { Cooked as a meal } \\
\text { Used to cook pastry }\end{array}$ \\
\hline Rumex tuberosus L. (ISTE 109881) & Ekşi kulak, Kuzukulağ1 & Leaf & Freshly eaten as a salad \\
\hline \multicolumn{4}{|l|}{ Portulacaceae } \\
\hline Portulaca oleracea L. (E.Ö.49) & Semizlik otu, Temizlik otu & Aerial part & $\begin{array}{l}\text { Cooked as a meal } \\
\text { Prepared canned food }\end{array}$ \\
\hline \multicolumn{4}{|l|}{ Rosaceae } \\
\hline Crataegus microphylla K.Koch (ISTE 109914, 109916) & Alıç & Fruit & Eaten \\
\hline $\begin{array}{l}\text { Crataegus monogyna Jacq. (ISTE 109915, 109917, } \\
\text { 109942, 109896) }\end{array}$ & Alıç, Alıç gülü, yemişen & Fruit & $\begin{array}{l}\text { Eaten } \\
\text { Molasses }\end{array}$ \\
\hline $\begin{array}{l}\text { Prunus divaricata Ledeb. subsp. divaricata (Ledeb.) } \\
\text { Schneider (E.Ö.54) }\end{array}$ & Dağ eriği, Erik & Fruit & $\begin{array}{l}\text { Dried fruits eaten } \\
\text { Pickle }\end{array}$ \\
\hline $\begin{array}{l}\text { Prunus spinosa L. (ISTE 109935, 109907, 109909, } \\
\text { 109937, 109930) }\end{array}$ & Çakal eriği, Deli erik, Domuz eriği & Fruit & $\begin{array}{l}\text { Eaten } \\
\text { Jam }\end{array}$ \\
\hline Pyrus amygdaliformis Vill. (ISTE 109911, 109936 ) & Deli armut & Fruit & Eaten \\
\hline Pyrus elaeagnifolia Pall. (ISTE 109923, 109933) & $\begin{array}{l}\text { Ahlat, Aflat, Alfat, Çakal armudu, Geyik } \\
\text { elması, Üvez, Yabani armut }\end{array}$ & Fruit & $\begin{array}{l}\text { Pickle } \\
\text { Eaten }\end{array}$ \\
\hline
\end{tabular}




\begin{tabular}{|c|c|c|c|}
\hline Botanical name, family and specimen number & Local name in Savaştepe & Used parts & / Usage \\
\hline Rosa canina L. (ISTE 109938, 109931) & $\begin{array}{|lll|}\begin{array}{l}\text { Bayırgülü, Iprım, İtgülü, } \\
\text { Kuşburnu }\end{array} & \text { Kuşbaşı, } \\
\end{array}$ & Fruit & Jam \\
\hline $\begin{array}{l}\text { Rosa sempervirens L. (ISTE 109902, 109901, 109906, } \\
\text { 109905, 109908) }\end{array}$ & Kuşburnu, İtgülü, Öküzgötü & Fruit & Jam \\
\hline Rubus canescens DC. (ISTE 109926) & Böğürtlen, Karamık, Karantı, Kırantı & Fruit & $\begin{array}{l}\text { Eaten } \\
\text { Jam }\end{array}$ \\
\hline $\begin{array}{l}\text { Rubus sanctus Schreb. (ISTE 109899, 109900, 109928, } \\
\text { 109929, 109939) }\end{array}$ & Böğürtlen, Kırıntı & Fruit & Eaten \\
\hline Sarcopoterium spinosum (L.) Spach (E.Ö.56) & $\begin{array}{l}\text { Çıtırdak, Çıtırgan, Çıtırık, Çıtırgı dikeni, } \\
\text { Çıtır pıtır }\end{array}$ & Aerial part & Eaten \\
\hline \multicolumn{4}{|l|}{\begin{tabular}{|l} 
Tiliaceae \\
\end{tabular}} \\
\hline Tilia tomentosa Moench (ISTE 109961) & Ihlamur & Leaf & Cooked as a meal (Sarma) \\
\hline \multicolumn{4}{|l|}{ Urticaceae } \\
\hline Urtica dioica L. (ISTE 109962, E.Ö.68) & $\begin{array}{l}\text { Bıcırgan, Gıcırgan, Gidişken, Isırgan, } \\
\text { Kabarcık }\end{array}$ & $\begin{array}{l}\text { Leaf } \\
\text { Aerial part, leaf }\end{array}$ & $\begin{array}{l}\text { Cooked as a meal } \\
\text { Used to cook pastry }\end{array}$ \\
\hline Urtica membranacea Poir. ex Savigny (ISTE 109964) & Isirgan & $\begin{array}{l}\text { Aerial part, leaf } \\
\text { Leaf }\end{array}$ & $\begin{array}{l}\text { Cooked as a meal } \\
\text { Used to cook pastry }\end{array}$ \\
\hline
\end{tabular}

Table 2: The comparison of uses of the same edible plants in Savaştepe (Balıkesir), Kırklareli, Inner-West Anatolia, İzmir, Madra Dağı, Marmara Island, Marmaris, Middle Aegean Region, Balıkesir region, Çatalca (—: plants not recorded)

\begin{tabular}{|c|c|c|c|c|c|c|c|c|c|c|}
\hline $\begin{array}{l}\text { Botanical name and } \\
\text { family }\end{array}$ & $\begin{array}{l}\text { Savaştepe } \\
\text { (Balıkesir) }\end{array}$ & Kurklareli & $\begin{array}{l}\text { Inner- } \\
\text { West } \\
\text { Anatolia }\end{array}$ & $\begin{array}{l}\text { İzmir local } \\
\text { market }\end{array}$ & $\begin{array}{l}\text { Madra } \\
\text { dağı } \\
\text { (izmir) }\end{array}$ & $\begin{array}{l}\text { Marmara } \\
\text { Island }\end{array}$ & Marmaris & \begin{tabular}{|l} 
Middle \\
Aegean \\
Region
\end{tabular} & \begin{tabular}{|l|} 
Balıkesir \\
region \\
edible \\
fruits \\
\end{tabular} & Çatalca \\
\hline $\begin{array}{l}\text { Anethum graveolens } \mathrm{L} . \\
\text { (Apiaceae) }\end{array}$ & Leaf; spice & - & - & \begin{tabular}{|l} 
Aerial \\
part; salad, \\
cooked \\
\end{tabular} & - & - & Leaf; spice & - & - & - \\
\hline $\begin{array}{l}\text { Arbutus andrachne L. } \\
\text { (Ericaceae) }\end{array}$ & $\begin{array}{l}\text { Fruit; fresh } \\
\text { eaten, jam, } \\
\text { molasses } \\
\end{array}$ & - & - & - & - & - & - & $\begin{array}{l}\text { Fruit; } \\
\text { eaten }\end{array}$ & Fruit; eaten & - \\
\hline $\begin{array}{l}\text { Capsella bursa- } \\
\text { pastoris (L.) Medik. } \\
\text { (Brassicaceae) } \\
\end{array}$ & $\begin{array}{l}\text { Aerial part; } \\
\text { cooked as a } \\
\text { meal, salad }\end{array}$ & - & - & - & - & - & - & $\begin{array}{l}\text { Aerial } \\
\text { part; } \\
\text { eaten }\end{array}$ & - & - \\
\hline $\begin{array}{l}\text { Carduus nutans L. } \\
\text { (Compositae) }\end{array}$ & Seed; eaten & $\begin{array}{l}\text { Stem; } \\
\text { after bark } \\
\text { is peeled } \\
\text { eaten }\end{array}$ & - & - & - & - & - & - & - & - \\
\hline $\begin{array}{l}\text { Chondrilla juncea L. } \\
\text { (Compositae) }\end{array}$ & $\begin{array}{l}\text { Aerial part; } \\
\text { cooked as a } \\
\text { meal }\end{array}$ & - & - & - & - & - & - & $\begin{array}{l}\text { Aerial } \\
\text { part; } \\
\text { eaten }\end{array}$ & - & - \\
\hline $\begin{array}{l}\text { Cornus mas L. } \\
\text { (Cornaceae) }\end{array}$ & $\begin{array}{l}\text { Fruit; jam, } \\
\text { compote }\end{array}$ & \begin{tabular}{|l|} 
Fruit; eaten \\
fresh or \\
in jam or \\
marmalade \\
\end{tabular} & \begin{tabular}{|l|} 
Fruit; eaten \\
fresh or \\
in jam or \\
marmalade \\
\end{tabular} & - & - & - & - & - & Fruit; eaten & Fruit; eaten \\
\hline $\begin{array}{l}\text { Crataegus microphylla } \\
\text { K.Koch (Rosaceae) }\end{array}$ & Fruit; eaten & - & - & - & - & - & - & - & Fruit; eaten & - \\
\hline $\begin{array}{l}\text { Crataegus monogyna } \\
\text { Jacq. (Rosaceae) }\end{array}$ & $\begin{array}{l}\text { Fruit; eaten, } \\
\text { molasses }\end{array}$ & - & Fruit; jam & - & $\begin{array}{l}\text { Fruit; } \\
\text { eaten }\end{array}$ & - & - & - & Fruit; eaten & - \\
\hline
\end{tabular}




\begin{tabular}{|c|c|c|c|c|c|c|c|c|c|c|}
\hline $\begin{array}{l}\text { Botanical name and } \\
\text { family }\end{array}$ & $\begin{array}{l}\text { Savaştepe } \\
\text { (Balıesir) }\end{array}$ & Kurklareli & $\begin{array}{l}\text { Inner- } \\
\text { West } \\
\text { Anatolia }\end{array}$ & $\begin{array}{l}\text { İzmir local } \\
\text { market }\end{array}$ & $\begin{array}{l}\text { Madra } \\
\text { dağı } \\
\text { (izmir) }\end{array}$ & $\begin{array}{l}\text { Marmara } \\
\text { Island }\end{array}$ & Marmaris & $\begin{array}{l}\text { Middle } \\
\text { Aegean } \\
\text { Region }\end{array}$ & $\begin{array}{l}\text { Balıkesir } \\
\text { region } \\
\text { edible } \\
\text { fruits } \\
\end{array}$ & Çatalca \\
\hline $\begin{array}{l}\text { Dioscorea communis } \\
\text { (L.) Caddick \& Wilkin } \\
\text { (Dioscoreaceae) }\end{array}$ & $\begin{array}{l}\text { Young shoot; } \\
\text { cooked with } \\
\text { egg, as a } \\
\text { omlet, freshly } \\
\text { eaten }\end{array}$ & - & - & $\begin{array}{l}\text { Fresh } \\
\text { shoots; } \\
\text { fried with } \\
\text { onion, egg }\end{array}$ & $\begin{array}{l}\text { Young } \\
\text { shoots; } \\
\text { eaten }\end{array}$ & - & - & - & - & - \\
\hline $\begin{array}{l}\text { Erodium cicutarium } \\
\text { (L.) L'Hér. } \\
\text { (Geraniaceae) } \\
\end{array}$ & $\begin{array}{l}\text { Aerial part; } \\
\text { freshly eaten } \\
\text { as a salad }\end{array}$ & - & $\begin{array}{l}\text { Aerial part; } \\
\text { eaten }\end{array}$ & $\begin{array}{l}\text { Aerial part; } \\
\text { cooked } \\
\text { pastry }\end{array}$ & - & - & - & $\begin{array}{l}\text { Aerial } \\
\text { part; } \\
\text { eaten }\end{array}$ & - & - \\
\hline $\begin{array}{l}\text { Ficus carica L. } \\
\text { (Moraceae) }\end{array}$ & Fruit; Jam & - & - & Fruit; jam & - & $\begin{array}{l}\text { Fruit; raw } \\
\text { eaten, jam }\end{array}$ & - & - & Fruit; eaten & - \\
\hline $\begin{array}{l}\text { Foeniculum vulgare } \\
\text { Mill. (Apiaceae) }\end{array}$ & Leaf; Spice & - & - & Leaf; spice & $\begin{array}{l}\text { Leaf; } \\
\text { eaten }\end{array}$ & - & - & $\begin{array}{l}\text { Aerial } \\
\text { part; } \\
\text { eaten }\end{array}$ & - & - \\
\hline $\begin{array}{l}\text { Lactuca serriola L. } \\
\text { (Compositae) }\end{array}$ & $\begin{array}{l}\text { Aerial part; } \\
\text { freshly eaten }\end{array}$ & - & - & - & - & $\begin{array}{l}\text { Leaf, } \\
\text { cooked; } \\
\text { fruit, raw } \\
\end{array}$ & - & $\begin{array}{l}\text { Aerial } \\
\text { part; } \\
\text { eaten }\end{array}$ & - & - \\
\hline $\begin{array}{l}\begin{array}{l}\text { Laurus nobilis L. } \\
\text { (Lauraceae) }\end{array} \\
\end{array}$ & Leaf; Spice & - & - & - & - & - & Leaf; spice & - & - & - \\
\hline $\begin{array}{l}\text { Leontodon tuberosus L. } \\
\text { (Compositae) }\end{array}$ & $\begin{array}{l}\text { Leaf; as a } \\
\text { salad eaten, } \\
\text { cooked as a } \\
\text { meal } \\
\end{array}$ & - & - & - & - & - & $\begin{array}{l}\text { Fresh leaf; } \\
\text { cooked as a } \\
\text { meal }\end{array}$ & - & - & - \\
\hline $\begin{array}{l}\text { Malva sylvestris L. } \\
\text { (Malvaceae) }\end{array}$ & \begin{tabular}{|l|} 
Leaf; cooked \\
as a meal, \\
cooked with \\
egg, as a \\
omlet, mixed \\
with cheese, \\
Used to cook \\
pastry \\
\end{tabular} & $\begin{array}{l}\text { Leaf; } \\
\text { cooked }\end{array}$ & - & $\begin{array}{l}\text { Leaf; } \\
\text { cooked } \\
\text { with meat, } \\
\text { pastry }\end{array}$ & - & $\begin{array}{l}\text { Leaf; } \\
\text { cooked, } \\
\text { sarma }\end{array}$ & $\begin{array}{l}\text { Aerial part; } \\
\text { cooked as a } \\
\text { meal }\end{array}$ & $\begin{array}{l}\text { Aerial } \\
\text { part; } \\
\text { eaten }\end{array}$ & - & - \\
\hline $\begin{array}{l}\text { Mentha longifolia (L.) } \\
\text { L. subsp. typhoides } \\
\text { (Briq.) Harley } \\
\text { (Lamiaceae) } \\
\end{array}$ & Leaf; Spice & - & Leaf; spice & - & - & - & - & $\begin{array}{l}\text { Aerial } \\
\text { part; } \\
\text { eaten }\end{array}$ & - & - \\
\hline $\begin{array}{l}\text { Morus nigra L. } \\
\text { (Moraceae) }\end{array}$ & $\begin{array}{l}\text { Fruit; } \\
\text { jam,eaten, } \\
\text { sherbet for } \\
\text { salads } \\
\end{array}$ & $\begin{array}{l}\text { Leaf; } \\
\text { cooked } \\
\text { Fruit; fresh } \\
\text { eaten }\end{array}$ & - & - & $\begin{array}{l}\text { Fruit; } \\
\text { eaten }\end{array}$ & - & \begin{tabular}{l|} 
Leaf; \\
cooked \\
Fruit; eaten
\end{tabular} & $\begin{array}{l}\text { Fruit; } \\
\text { eaten }\end{array}$ & - & $\begin{array}{l}\text { Branches; } \\
\text { peeled, } \\
\text { slices, } \\
\text { eaten }\end{array}$ \\
\hline $\begin{array}{l}\text { Origanum vulgare L. } \\
\text { subsp. hirtum (Link) } \\
\text { Ietsw. (Lamiaceae) }\end{array}$ & $\begin{array}{l}\text { Aerial part; } \\
\text { mixed with } \\
\text { cheese, used } \\
\text { to cook } \\
\text { pastry, spice } \\
\end{array}$ & - & $\begin{array}{l}\text { Aerial part; } \\
\text { spice }\end{array}$ & - & - & $\begin{array}{l}\text { Aerial } \\
\text { part; spice }\end{array}$ & - & - & - & - \\
\hline $\begin{array}{l}\text { Papaver somniferum L. } \\
\text { (Papaveraceae) }\end{array}$ & $\begin{array}{l}\text { Seed oil; used } \\
\text { for cooking }\end{array}$ & - & \begin{tabular}{|l|} 
Seed; \\
katmer \\
cooking; \\
leaf salad \\
\end{tabular} & - & - & - & - & - & - & - \\
\hline $\begin{array}{l}\text { Pinus brutia Ten. } \\
\text { (Pinaceae) }\end{array}$ & $\begin{array}{l}\text { Resin; mixed } \\
\text { with honey } \\
\text { and eaten }\end{array}$ & - & - & - & $\begin{array}{l}\text { Resin; } \\
\text { eaten }\end{array}$ & - & - & - & - & - \\
\hline
\end{tabular}




\begin{tabular}{|c|c|c|c|c|c|c|c|c|c|c|}
\hline $\begin{array}{l}\text { Botanical name and } \\
\text { family }\end{array}$ & $\begin{array}{l}\text { Savaştepe } \\
\text { (Balıkesir) }\end{array}$ & Kırklareli & $\begin{array}{l}\text { Inner- } \\
\text { West } \\
\text { Anatolia }\end{array}$ & $\begin{array}{l}\text { İzmir local } \\
\text { market }\end{array}$ & $\begin{array}{l}\text { Madra } \\
\text { dağı } \\
\text { (izmir) }\end{array}$ & $\begin{array}{l}\text { Marmara } \\
\text { Island }\end{array}$ & Marmaris & \begin{tabular}{|l} 
Middle \\
Aegean \\
Region
\end{tabular} & \begin{tabular}{|l|} 
Balıkesir \\
region \\
edible \\
fruits \\
\end{tabular} & Çatalca \\
\hline $\begin{array}{l}\text { Pistacia terebinthus L. } \\
\text { (Anacardiaceae) }\end{array}$ & $\begin{array}{l}\text { Dry fruit; as } \\
\text { coffea,Young } \\
\text { leaf; eaten } \\
\text { fresh, Oil } \\
\text { (From fruit); } \\
\text { used to cook } \\
\text { pastry, fruit; } \\
\text { eaten, pickle }\end{array}$ & - & - & $\begin{array}{l}\text { Young } \\
\text { shoot; } \\
\text { eaten, } \\
\text { added to } \\
\text { pickle, } \\
\text { fried with } \\
\text { egg }\end{array}$ & $\begin{array}{l}\text { Fruit; } \\
\text { eaten }\end{array}$ & $\begin{array}{l}\text { Shoot; } \\
\text { salad }\end{array}$ & $\begin{array}{l}\text { Leaf; } \\
\text { boiled as a } \\
\text { meal, Fruit; } \\
\text { eaten }\end{array}$ & $\begin{array}{l}\text { Fruit; } \\
\text { eaten }\end{array}$ & Fruit; eaten & - \\
\hline $\begin{array}{l}\text { Polygonum cognatum } \\
\text { Meisn. (Polygonaceae) }\end{array}$ & $\begin{array}{l}\text { Aerial part; } \\
\text { cooked as a } \\
\text { meal, used to } \\
\text { cook pastry }\end{array}$ & - & $\begin{array}{l}\text { Aerial part; } \\
\text { eaten }\end{array}$ & $\begin{array}{l}\text { Aerial part; } \\
\text { fried with } \\
\text { egg, made } \\
\text { into soup } \\
\text { with wheat } \\
\text { grains, } \\
\text { prepared } \\
\text { as pastry } \\
\end{array}$ & - & - & - & $\begin{array}{l}\text { Aerial } \\
\text { part; } \\
\text { eaten }\end{array}$ & - & - \\
\hline $\begin{array}{l}\text { Portulaca oleracea L. } \\
\text { (Portulacaceae) }\end{array}$ & $\begin{array}{l}\text { Aerial part; } \\
\text { cooked } \\
\text { as a meal, } \\
\text { prepared } \\
\text { canned food }\end{array}$ & $\begin{array}{l}\text { Aerial part; } \\
\text { cooked }\end{array}$ & $\begin{array}{l}\text { Aerial part; } \\
\text { eaten }\end{array}$ & $\begin{array}{l}\text { Aerial } \\
\text { part; salad, } \\
\text { cooked }\end{array}$ & - & - & - & - & - & - \\
\hline $\begin{array}{l}\text { Prunus divaricata } \\
\text { Ledeb. subsp. } \\
\text { divaricata (Ledeb.) } \\
\text { Schneider (Rosaceae) }\end{array}$ & $\begin{array}{l}\text { Dried fruits; } \\
\text { eaten, pickle }\end{array}$ & \begin{tabular}{|l|} 
Fruit; jam, \\
marmalade, \\
compote
\end{tabular} & - & - & - & - & - & $\begin{array}{l}\text { Fruit; } \\
\text { eaten }\end{array}$ & Fruit; eaten & - \\
\hline $\begin{array}{l}\text { Prunus spinosa L. } \\
\text { (Rosaceae) }\end{array}$ & $\begin{array}{l}\text { Fruit; Eaten, } \\
\text { Jam }\end{array}$ & \begin{tabular}{|l|} 
Fruit; \\
pickle, jam, \\
marmalade, \\
compote
\end{tabular} & - & - & - & - & - & $\begin{array}{l}\text { Fruit; } \\
\text { eaten }\end{array}$ & Fruit; eaten & Fruit; jam \\
\hline $\begin{array}{l}\text { Pyrus amygdaliformis } \\
\text { Vill. (Rosaceae) }\end{array}$ & Fruit; eaten & - & - & - & $\begin{array}{l}\text { Fruit; } \\
\text { eaten }\end{array}$ & $\begin{array}{l}\text { Fruit; } \\
\text { pickle }\end{array}$ & Fruit; eaten & & Fruit; eaten & \\
\hline $\begin{array}{l}\text { Pyrus elaeagnifolia } \\
\text { Pall. (Rosaceae) }\end{array}$ & $\begin{array}{l}\text { Fruit; pickle, } \\
\text { eaten }\end{array}$ & Fruit; pickle & $\begin{array}{l}\text { Fruit; } \\
\text { pickle }\end{array}$ & - & - & - & - & $\begin{array}{l}\text { Fruit; } \\
\text { eaten }\end{array}$ & Fruit; eaten & $\begin{array}{l}\text { Fruit; } \\
\text { marmalade }\end{array}$ \\
\hline $\begin{array}{l}\text { Rapistrum rugosum } \\
\text { (L.) All. (Brassicaceae) }\end{array}$ & $\begin{array}{l}\text { Leaf; cooked } \\
\text { with egg, as a } \\
\text { omlet, freshly } \\
\text { eaten }\end{array}$ & - & - & - & - & - & - & $\begin{array}{l}\text { Aerial } \\
\text { part; } \\
\text { eaten }\end{array}$ & - & - \\
\hline $\begin{array}{l}\text { Rhus coriaria L. } \\
\text { (Anacardiaceae) }\end{array}$ & $\begin{array}{l}\text { Fruit, seed; } \\
\text { spice, Seed; } \\
\text { powdered } \\
\text { and used in } \\
\text { pastry, Fruit, } \\
\text { stem; used } \\
\text { in vinegar, } \\
\text { pickle } \\
\end{array}$ & - & - & - & - & Fruit; spice & - & - & - & - \\
\hline $\begin{array}{l}\text { Rosa canina L. } \\
\text { (Rosaceae) }\end{array}$ & Fruit; jam & - & Fruit; jam & - & $\begin{array}{l}\text { Fruit; } \\
\text { eaten }\end{array}$ & Fruit; jam & - & $\begin{array}{l}\text { Fruit; } \\
\text { eaten }\end{array}$ & Fruit; eaten & - \\
\hline
\end{tabular}




\begin{tabular}{|c|c|c|c|c|c|c|c|c|c|c|}
\hline $\begin{array}{l}\text { Botanical name and } \\
\text { family }\end{array}$ & $\begin{array}{l}\text { Savaştepe } \\
\text { (Balıkesir) }\end{array}$ & Kırklareli & $\begin{array}{l}\text { Inner- } \\
\text { West } \\
\text { Anatolia }\end{array}$ & $\begin{array}{l}\text { İzmir local } \\
\text { market }\end{array}$ & $\begin{array}{l}\text { Madra } \\
\text { dağı } \\
\text { (izmir) }\end{array}$ & $\begin{array}{l}\text { Marmara } \\
\text { Island }\end{array}$ & Marmaris & $\begin{array}{l}\text { Middle } \\
\text { Aegean } \\
\text { Region }\end{array}$ & \begin{tabular}{|l|} 
Balıkesir \\
region \\
edible \\
fruits \\
\end{tabular} & Çatalca \\
\hline $\begin{array}{l}\text { Rosmarinus officinalis } \\
\text { L. (Lamiaceae) }\end{array}$ & Leaf; spice & - & - & - & - & - & Leaf; spice & - & - & - \\
\hline $\begin{array}{l}\text { Rubus canescens DC. } \\
\text { (Rosaceae) }\end{array}$ & $\begin{array}{l}\text { Fruit; eaten, } \\
\text { jam }\end{array}$ & $\begin{array}{l}\text { Fruit; } \\
\text { fresh, jam, } \\
\text { marmalade } \\
\end{array}$ & Fruit; jam & - & - & - & - & $\begin{array}{l}\text { Fruit; } \\
\text { eaten }\end{array}$ & Fruit; eaten & - \\
\hline $\begin{array}{l}\text { Rubus sanctus Schreb. } \\
\text { (Rosaceae) }\end{array}$ & Fruit; eaten & - & - & - & $\begin{array}{l}\text { Fruit; } \\
\text { eaten }\end{array}$ & $\begin{array}{l}\text { Fruit; raw, } \\
\text { jam }\end{array}$ & $\begin{array}{l}\text { Fruit; eaten } \\
\text { fresh }\end{array}$ & $\begin{array}{l}\text { Fruit; } \\
\text { eaten }\end{array}$ & Fruit; eaten & Fruit; eaten \\
\hline $\begin{array}{l}\text { Rumex acetosella L. } \\
\text { (Polygonaceae) }\end{array}$ & \begin{tabular}{|l} 
Seed; eaten, \\
Leaf; Freshly \\
eaten as a \\
salad, cooked \\
as a meal \\
\end{tabular} & $\begin{array}{l}\text { Leaf; } \\
\text { cooked }\end{array}$ & - & $\begin{array}{l}\text { Aerial; } \\
\text { salad }\end{array}$ & $\begin{array}{l}\text { Leaf; } \\
\text { eaten }\end{array}$ & $\begin{array}{l}\text { Young } \\
\text { shoots; } \\
\text { raw in } \\
\text { salad }\end{array}$ & - & - & - & - \\
\hline $\begin{array}{l}\text { Rumex crispus L. } \\
\text { (Polygonaceae) }\end{array}$ & \begin{tabular}{|l|} 
Leaf; Cooked \\
as a meal \\
\end{tabular} & \begin{tabular}{|l|}
$\begin{array}{l}\text { Leaf; } \\
\text { cooked }\end{array}$ \\
\end{tabular} & Leaf; eaten & - & - & - & - & - & - & - \\
\hline $\begin{array}{l}\text { Rumex patientia L. } \\
\text { (Polygonaceae) }\end{array}$ & $\begin{array}{l}\text { Leaf; Cooked } \\
\text { as a meal, } \\
\text { used to cook } \\
\text { pastry }\end{array}$ & - & - & $\begin{array}{l}\text { Leaf; fried } \\
\text { with egg, } \\
\text { onion; } \\
\text { cooked as } \\
\text { a pastry; } \\
\text { sarma }\end{array}$ & $\begin{array}{l}\text { Leaf; } \\
\text { eaten }\end{array}$ & - & - & $\begin{array}{l}\text { Leaf; } \\
\text { eaten }\end{array}$ & - & - \\
\hline $\begin{array}{l}\text { Rumex tuberosus L. } \\
\text { (Polygonaceae) }\end{array}$ & $\begin{array}{l}\text { Leaf; Freshly } \\
\text { eaten as a } \\
\text { salad }\end{array}$ & - & - & - & - & - & Leaf; eaten & - & - & - \\
\hline $\begin{array}{l}\text { Ruscus aculeatus L. } \\
\text { (Asparagaceae) }\end{array}$ & Fruit; Eaten & - & - & - & - & - & - & - & - & Fruit; eaten \\
\hline $\begin{array}{l}\text { Sonchus asper (L.) } \\
\text { Hill subsp. glaucescens } \\
\text { (Jord.) Ball ex Ball } \\
\text { (Compositae) }\end{array}$ & $\begin{array}{l}\text { Aerial part; } \\
\text { cooked as a } \\
\text { meal, freshly } \\
\text { eaten }\end{array}$ & - & - & \begin{tabular}{|l|} 
Leaf; \\
fried with \\
onion, egg; \\
cooked as \\
a pastry \\
\end{tabular} & $\begin{array}{l}\text { Leaf; } \\
\text { eaten }\end{array}$ & - & - & $\begin{array}{l}\text { Aerial } \\
\text { part; } \\
\text { eaten }\end{array}$ & - & - \\
\hline $\begin{array}{l}\text { Thymus longicaulis } \\
\text { C.Presl subsp. } \\
\text { chaubardii (Rchb.f.) } \\
\text { Jalas (Lamiaceae) } \\
\end{array}$ & $\begin{array}{l}\text { Aerial part; } \\
\text { spice }\end{array}$ & - & $\begin{array}{l}\text { Aerial part; } \\
\text { spice }\end{array}$ & - & - & Leaf; spice & - & - & - & - \\
\hline $\begin{array}{l}\text { Urtica dioica L. } \\
\text { (Urticaceae) }\end{array}$ & \begin{tabular}{|l} 
Aerial part, \\
leaf; cooked \\
as a meal, \\
used to cook \\
pastry \\
\end{tabular} & $\begin{array}{l}\begin{array}{l}\text { Leaf, } \\
\text { cooked }\end{array} \\
\end{array}$ & $\begin{array}{l}\text { Aerial part; } \\
\text { eaten }\end{array}$ & - & $\begin{array}{l}\text { Aerial } \\
\text { part; } \\
\text { eaten }\end{array}$ & $\begin{array}{l}\text { Leaf; } \\
\text { cooked }\end{array}$ & - & $\begin{array}{l}\text { Aerial } \\
\text { part; } \\
\text { eaten }\end{array}$ & - & - \\
\hline $\begin{array}{l}\text { Urtica membranacea } \\
\text { Poir. ex Savigny } \\
\text { (Urticaceae) }\end{array}$ & $\begin{array}{l}\text { Aerial } \\
\text { part, leaf; } \\
\text { cooked as a } \\
\text { meal,used to } \\
\text { cook pastry } \\
\end{array}$ & - & - & - & - & - & $\begin{array}{l}\text { Aerial part; } \\
\text { cooked as a } \\
\text { meal }\end{array}$ & - & - & - \\
\hline
\end{tabular}




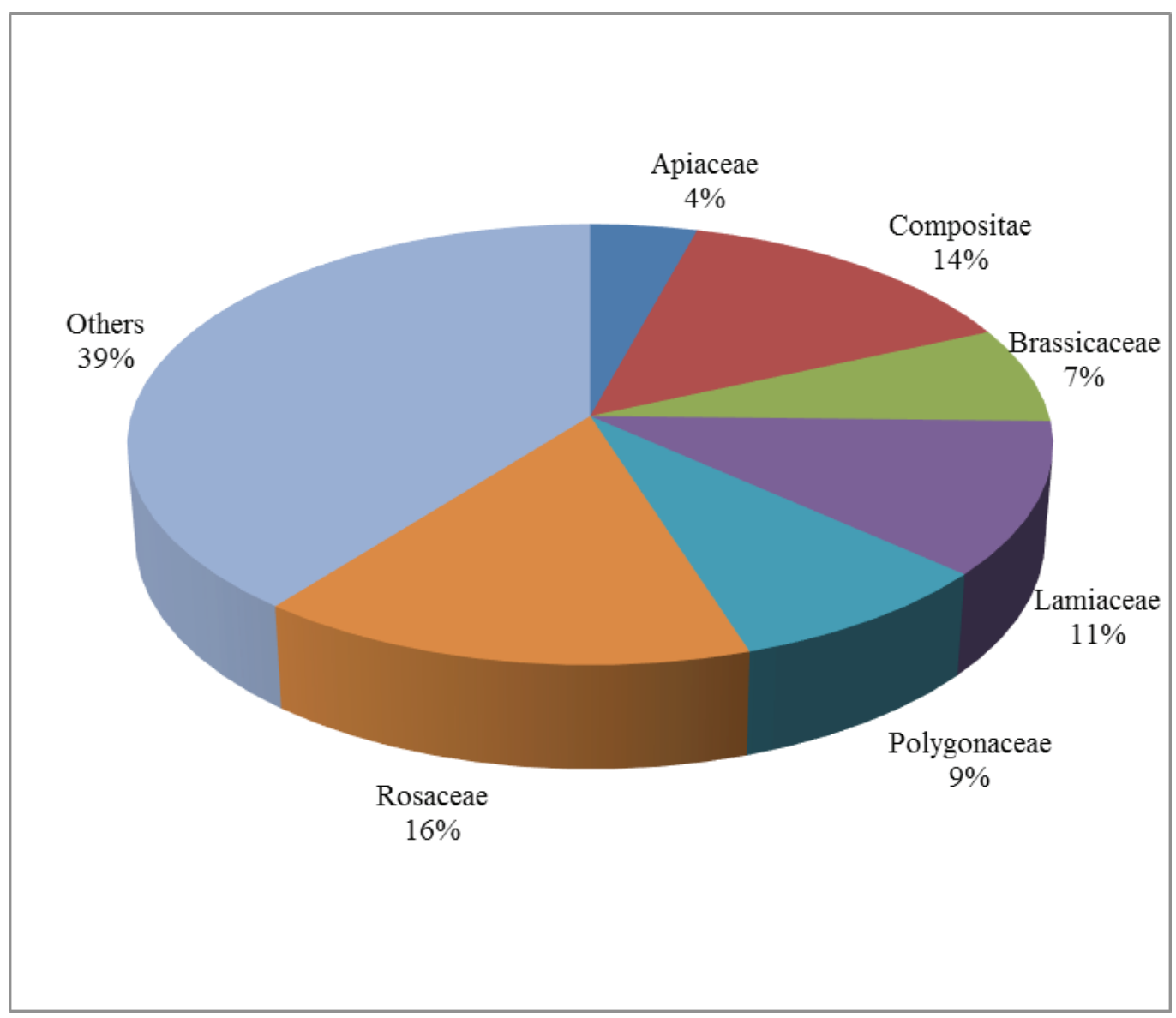

Figure 6. The most frequently used families

\section{Acknowledgements}

This research is the part of the $\mathrm{PhD}$ thesis "An Ethnobotanical Study in Savaştepe and Kepsut Region (Balıkesir)" that was supported by Istanbul University, Scientific Research Project Unit. Project No: 41370

\section{Savaştepe (Balıkesir, Türkiye) İlçesinin Yabani Besin Bitkiler}

\section{ÖZ}

Bu etnobotanik çalsşma Savaştepe ilçesinde (Balıkesir), Mayıs 2012-Ekim 2015 tarihleri arasında gerçekleştirildi. Savaştepe'de bulunan yerleşim yerleri (44 köy) arazi çalışmaları sırasında ziyaret edildi. Bu çalışmayla, Savaştepe'de (Balıkesir) besin olarak kullanılan bitkilerin geleneksel kullanımlarının belgelenmesi hedeflendi. Yöre halkı ile yüzyüze görüşmeler yapıldı. Bölgede toplam 205 kişi (107 erkek, 98 kadın) ile görüşüldü. Bitki örnekleri yerel halk ile birlikte topland. Toplanan türler tayin edildi ve ISTE’de (İstanbul Üniversitesi Eczacllı Fakültesi Herbaryumu) herbaryum örnekleri haline getirilerek saklandi. Toplanan bitkiler arasinda 27 familyaya ait 71 taksonun besin amaçlı tüketildiği kaydedilmiştir. Bitkiler çoğunlukla gıda (55 takson), baharat (14 takson), içecek (3 takson) ve ballı macun (2 takson) şeklinde kullanılmaktadır. Çoğunlukla kullanılan bitki bölümleri yaprak (27 takson), toprak üstü kısım (20 takson), meyve (19 takson) ve tohum (5 takson) olarak tespit edildi.

Anahtar kelimeler: Etnobotanik, Yabani besin bitkileri, Savaştepe, Balıkesir, Türkiye 


\section{References:}

1. Güner A, Aslan S, Ekim T, Vural M, Babaç MT. Türkiye Bitkileri Listesi (Damarlı Bitkiler). Nezahat Gökyiğit Botanik Bahçesi ve Flora Araştırmaları Derneği Yayını. İstanbul. 2012.

2. Özdemir Nath E. An Ethnobotanical Study in Savaştepe and Kepsut Region (Balıkesir). Istanbul University, Institute of Health Science, Department of Pharmaceutical Botany. Phd. Thesis. Istanbul. 2016. (Supervisor : Prof. Dr. Şükran Kültür)

3. Davis PH. Flora of Turkey and the East Aegean Islands, Vol 1-9. Edinburgh University Press, Edinburgh. 1965-1985.

4. Davis PH, Mill RR, Tan K. Flora of Turkey and the East Aegean Islands, Vol 10. Edinburgh University Press, Edinburgh. 1998.

5. Güner A, Özhatay N, Ekim T, Başer KHC. Flora of Turkey and the East Aegean Islands, Vol 11. Edinburgh University Press, Edinburgh. 2000.

6. http://www.theplantlist.org/tpl1.1/record/kew-2517619

7. Gürdal B, Kültür Ş. The edible and miscellaneous useful plants in Marmaris (Southwest Turkey). J Fac Pharm Istanbul Univ 2014; 44:69-78.

8. Bulut G. Medicinal and wild food plants of Marmara Island (Balikesir-Turkey). Acta Soc Bot Pol 2016; 85:3501-16.

9. Dogan Y, Ugulu I, Durkan N. Wild edible plants sold in the local markets of Izmir, Turkey. Pak J Bot 2013; 45:177-84.

10. Duran A, Satıl F, Tümen G. Balıkesir Yöresinde yenen yabani meyveler ve etnobotanik özellikleri. Ot Sistematik Botanik Derg 2001; 8:87-94.

11. Ecevit Genç G, Özhatay N. An ethnobotanical study in Çatalca (European part of İstanbul) II. Turk J Pharm Sci 2006; 3:7389.

12. Kargıoğlu M, Cenkci S, Serteser A, Evliyaoğlu N, Konuk M, Kök MŞ, Bağcı Y. An ethnobotanical survey of inner-West Anatolia, Turkey. Hum Ecol 2008; 36:763-77.

13. Kargıoğlu M, Cenkci S, Serteser A, Konuk M, Vural G. Traditional uses of wild plants in the middle Aegean region of Turkey. Hum Ecol 2010; 38:429-50.

14. Kültür Ş. An ethnobotanical study of Kırklareli (Turkey). Phytol Balc 2008; 14:279-89.

15. Satıl F, Akçiçek E, Selvi S. Madra Dağı (Balıkesir/İzmir) ve çevresinde etnobotanik bir çalışma. Biyoloji Bilimleri Araştırma Derg 2008; 1:31-6.

16. Tuzlacı E. Türkiye'nin yabani besin bitkileri ve ot yemekleri. Alfa Yayınları, İstanbul. 2011.

17. Özdemir E, Kültür Ş. Some medicinal food plants of Savaştepe District (Balıkesir, Turkey). 2nd IVEK International Convention of Pharmaceuticals and Pharmacies. 2015; 110. 